\title{
EDITORIAL
}

Adv Clin Exp Med 2015, 24, 4, 555-561

(C) Copyright by Wroclaw Medical University

DOI: $10.17219 /$ acem/31239

ISSN 1899-5276

Łukasz Szylberg ${ }^{1, A-C, E}$, Marlena Janiczek ${ }^{1, B-D}$, Aneta Popiel $^{1, B-D}$, ANDRZEJ MARSZAŁEK 2 , E, F

\section{Large Bowel Genetic Background and Inflammatory Processes in Carcinogenesis - Systematic Review}

\author{
${ }^{1}$ Department of Clinical Pathomorphology, Collegium Medicum in Bydgoszcz, Nicolaus Copernicus University \\ in Toruń, Poland \\ 2 Department of Oncological Pathology, Poznan University of Medical Sciences and Greater Poland Oncology \\ Center, Poland
}

A - research concept and design; $\mathbf{B}$ - collection and/or assembly of data; C - data analysis and interpretation;

$\mathbf{D}$ - writing the article; $\mathbf{E}$ - critical revision of the article; $\mathbf{F}$ - final approval of article; $\mathbf{G}$ - other

\begin{abstract}
Colorectal cancer (CRC) has become the third most common cancer in developed countries. Each year more and more people die from CRC. CRC is also one of the most effectively studied topics in recent years. It has been found that the key phenomena in CRC development are genetic and inflammatory processes. Well-known genetic bases for the carcinogenesis of CRC include chromosomal changes characteristic of the chromosomal instability pathway which correlates with specific and well-defined genetic alterations (such as APC, K-RAS, DCC and p53) and genomic instability characteristics for the mutator pathway focused on KRAS and BRAF mutations. Recent studies have highlighted the impact of inflammation in CRC, especially elevated levels of pro-inflammatory cytokines. Among important risk factors of colon carcinogenesis are colorectal polyps, which are currently the subject of intense research. Recent studies have shown that different adenomas are characterized by different pathways of carcinogenesis as well as diverse COX-2 expression in various polyps. Understanding the mechanism of inflammatory processes in CRC parallel to basic genetic alterations might allow for effective and targeted treatment (Adv Clin Exp Med 2015, 24, 4, 555-561).
\end{abstract}

Key words: colorectal cancer, inflammation, carcinogenesis, genetic alterations.

Colorectal cancer (CRC) has become the third most common cancer in developed countries [1]. CRC is also one of the most effectively studied topics in recent years. The pathogenesis of CRC is still not fully understood. Initially it was thought that the key role in its pathogenesis involved genetic mutations. A number of studies have been performed to confirm this hypothesis. Karoliina Stefanius' study shows two distinct ways of CRC carcinogenesis, depending on the existing mutation [2]. Although there is a well-known genetic basis for the carcinogenesis of colorectal cancer, this knowledge does not allow us to introduce new, effective CRC treatment modalities.

In recent decades, many studies have highlighted the impact of inflammation in CRC. In 1863, Virchow observed a relationship between cancer and chronic inflammation [3]. Recently, the effects of the inflammatory process on neoplasia development were demonstrated in endometrial, cervical, ovarian, breast, prostate and colon tumors [4]. Innate immune system cells are included in the microenvironment of tumors. These cells secrete proinflammatory cytokines, chemokines, growth factors and reactive oxygen species that could cause DNA damage $[5,6]$. The inflammatory process most likely has a key role in the pathogenesis of CRC, especially in its promotion. This is an important factor in cancer staging and prognosis. The aim of currently research is to discover the factor initiating the inflammatory process, which potentially could be used effectively for preventive activities. Another important aspect of research is focused on the determination of the exact time and 
location of the inflammation that leads to carcinogenesis. Most likely, understanding the mechanisms of inflammatory processes in CRC and combining it with a genetic background should allow for a reduction in the number of cases and for effective treatment.

Studies of CRC have been focused on sporadic and familial cases. Hereditary colon cancer predisposition is confirmed in less than $5 \%$ of all CRC patients. Detailed information in such cases provides the opportunity for isolation of the genetic, environmental, and epigenetic factors which determine the phenotypes of the cancer. An important phenomenon of colon carcinogenesis is its development preceded by colorectal adenomas, which are currently under intense research. According to the data published, almost half of the population will develop at least one benign adenomatous colon polyp during his or her lifetime $[7,8]$. Three percent of these patients will develop CRC. Adenomas are considered an important risk factor in CRC and the removal of adenomatous polyps has been shown to reduce the risk of development of CRC. The polyps of colon cancer are divided into 3 subgroups according to their malignant potential (Fig. 1). Namely, there are hyperplastic polyps (HPP) with the least malignant potential, serrated polyps/adenoma (SA) and adenoma with a high malignant potential. Adenomas are classified into 3 types according to their morphological nature. These are villous (VA), tubular (TA) and tubulovillous (TVA). An interesting subgroup of lesions are serrated adenomas, which are discussed in conjunction with specific conditions such as adenoma and hyperplastic polyps. The term serrated

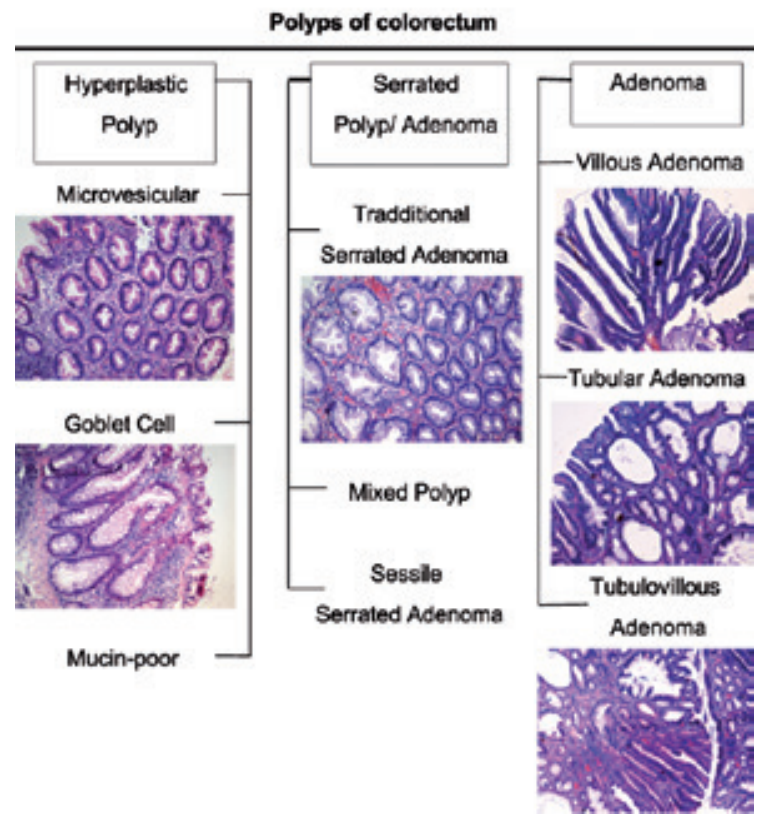

Fig. 1. Types of colon polyps adenoma was introduced by Longacre and Fenoglio-Preiser [9]. Serrated adenoma are divided into 3 subtypes. These are traditional serrated adenoma, sessile serrated adenoma (SSA) and mixed polyps (MP).

\section{Hyperplastic Polyps}

HP, typically are small, smooth, sessile lesions which are usually located in the distal colon and rectum and are diagnosed in patients above 40 years of age. The hyperplastic polyp has a lot of crypts with a convoluted luminal pattern and immature proliferative cells on the lower portion. Superficial serration could be observed. Regarding their cellular composition, polyps can be classified as microvesicular, goblet cells or mucin-poor variants.

\section{Traditional Serrated Adenoma}

These were described for the first time in 1984 as a group of lesions with features of both hyperplastic and adenomatous polyps and were thought as "mixed hyperplastic adenomatous polyps" [10]. Traditional serrated adenoma have a serrated architecture with characteristic cytological features of central, elongated nuclei, mild pseudostratification and eosinophilic cytoplasm and with microscopic impression of serration in crypts. In contrast to conventional adenomas, traditional serrated adenomas might present more hyperchromatic nuclei in the lower crypts than those on the surface and more basophilic cytoplasm. The common feature of TSAs and conventional adenomas is location. Eighty percent of TSAs are located in the left colon, mostly the rectosigmoid region $[10,11]$.

\section{Sessile Serrated Adenoma}

This term was recently introduced by Torlakovic and colleagues [10]. SSA is the premalignant sessile lesion of the colon with crypts without traditional dysplasia in contrast to adenomas or traditional serrated adenomas. The basal crypts are not compact and with architecture different from the hyperplastic polyps. The crypts usually are dilated on the bottom and narrowed in the middle part. The area of proliferation is not symmetrical and mitoses could be present in the upper part of the crypts. Generally SSA can be found in the right colon of middle-aged females $[10,11]$. 


\section{Traditional Adenoma}

This is a benign neoplasm of glandular origin lined by columnar epithelium without chief and parietal cells. Serration is not the dominant feature of traditional adenomas. The incidence of TA increases with age. According to published data, about $20-30 \%$ of patients with polyps lesions developed them under 40 years of age, while the occurrence of colorectal adenomas after 60 years of age increases to $40-50 \%$. There is no difference between the incidence between women and men. There is a family predisposition to the occurrence of adenomas. All such lesions arise from adenomatous proliferation of epithelial cells. Additionally, dysplasia could be found, which range from low to high grade, reflecting neoplastic transformation potential.

\section{Mixed Polyp}

This change has the elements of the structure of a typical hyperplastic polyp, and focal AT, AV, ATV or TSA.

\section{Influence of Genetic Mutation in Neoplasia of Colorectal Cancer}

Based on Fearon and Vogelstein's research, we can define the main multi-step process for CRC development as "the adenoma-carcinoma sequence" [12] (Fig. 2). Today we also call it the CIN (chromosomal instability) pathway, which correlates to specific genetic events within the involved tissue. The other two main pathways, in contrast to chromosomal changes, show genomic instability called MSI (microsatellite instability) - the process characteristic for mutator pathways.
It has been well proven in HNPCC (hereditary non-polyposis colorectal cancer) syndrome, in which the cancer is caused by a germ-line mutation of genes engaged in the mismatch repair (MMR) system. The mutator pathway it is also called the "serrated pathway" and related to sporadic CRCs, in which an aberration is present in MMR and in other DNA repair systems. These events are caused by hypermethylation. Tumors caused by the aforementioned pathway also show MSI [12]. In a classic "adenoma-carcinoma sequence", every step from the normal mucosa towards the carcinoma was attributed to specific and well-defined genetic alterations such as APC (adenomatous polyposis coli), oncogenes K-RAS, DCC (deleted in CRC) and p53. The first step includes a loss of APC suppressor gene function. APC contains 15 exons and it is mutated in $60 \%$ and $82 \%$ of colon and rectal cancers, respectively [13]. APC function is closely linked to $\beta$-catenin. Normally, it stimulates $\beta$-catenin destruction in proteasomes. If this gene is disrupted, it leads to $\beta$-catenin accumulation and translocation to the cell nucleus, where it activates transcription of several genes such as MYC and cyclin $\mathrm{D}$, which stimulate proliferation [2].

The next genetic alteration is RAS mutation. As K-RAS, it leads to disturbances in the genetic material, giving rise to cancerogenesis. Protooncogene located at $12 \mathrm{p} 12.1$, which encodes the 21-kDa GTP-binding protein, plays an important role in transmitting extracellular signals into an intracellular signal. Transduction mediates cellular responses to growth signals by switching between active GTP and inactive GTP forms of RAS mutation. Mutated RAS in its active form stimulates cells to mitosis and inhibits apoptosis of cells. RAS mutations are present with the same frequency in large adenomas and carcinomas, but with lower numbers in small adenomas. This might lead to the conclusion that RAS mutation is a late event in the "adenoma-carcinoma sequence" [14].

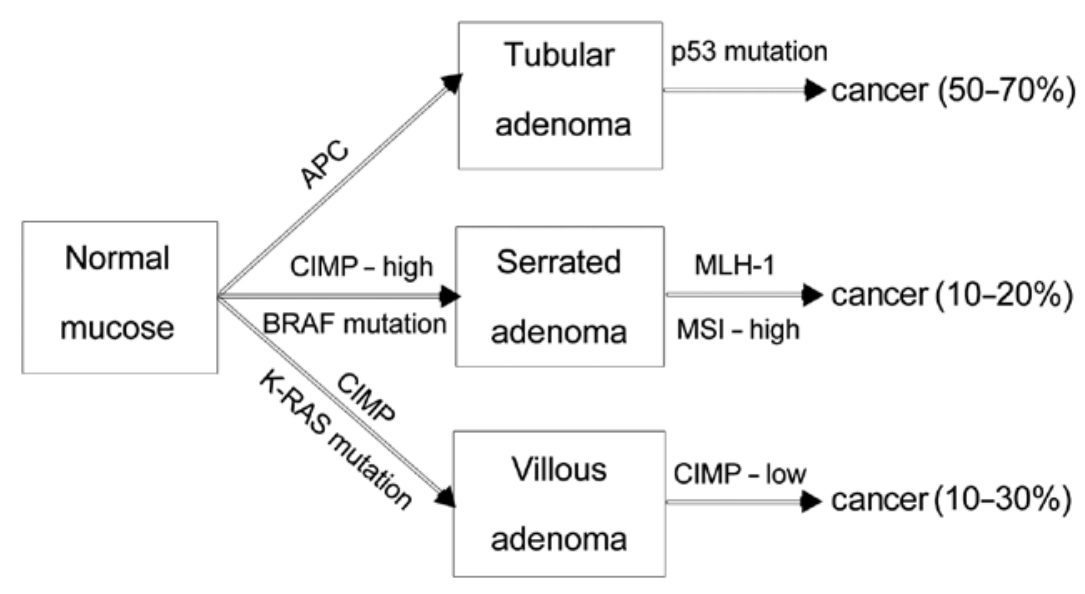

Fig. 2. The main multistep process for CRC development - "the adenoma-carcinoma sequence" 
Another genetic alteration leading to cancer is DCC, located at $18 \mathrm{q} 21.1$. About $60-70 \%$ of colorectal cancers show allelic losses in DCC. It is not fully understood how this gene is linked to cancerogenesis [15]. The study results point out that the wild-type DCC, but not the mutant one, induces apoptosis and activates caspase- 3 , and that DCC expression induces a rapid G2/M cell cycle arrest in some cell lines [2].

The most common mutation, present in 70 $-80 \%$, is loss of the function of TP 53 . Mutation of TP 53 usually occurs at the time of the transition from adenoma to cancer [15]. The frequency of TP 53 mutations have a propensity to increase with the progression of the lesion [13]. Thus changes are found in $4-26 \%$ of adenomas, $50 \%$ of adenomas with invasive foci, and in $50-75 \%$ of CRCs. The $\mathrm{P} 53$ protein induces $\mathrm{G} 1$ cell-cycle inhibition to facilitate DNA repair during the replication of cells exposed to environmental or oncogenic stress [2].

In recent years, several scientific projects have focused on the mutator pathway, which encloses 2 subtypes (Fig. 3), one characterized by K-RAS and the other one by BRAF mutation. However, the same feature is present, MSI, but at different levels. Microsatellite instability is a result of a defective MMR (mismatch repair) system that leads to slippage DNA polymerase during DNA replication. According to the level of MSI, the tumors differ in clinical and pathological features. There is MSI (microsatellite instability) at high (MSI-H) or low (MSI-L) levels. MSI-H is a result of a loss of DNA mismatch repair activity. MSI-L is a consequence of MMR deficiency. Of note is the correlation between the occurrence K-RAS mutation and MSI-L or BRAF mutation and the presence of MSI-H. The mutator pathway has two variants. One uses K-RAS mutations and the other BRAF mutations. A common feature for both is the presence of MSI. The mutator pathway presents a phenomen of the CpG-island methylator pathway (CIMP). Normally, DNA

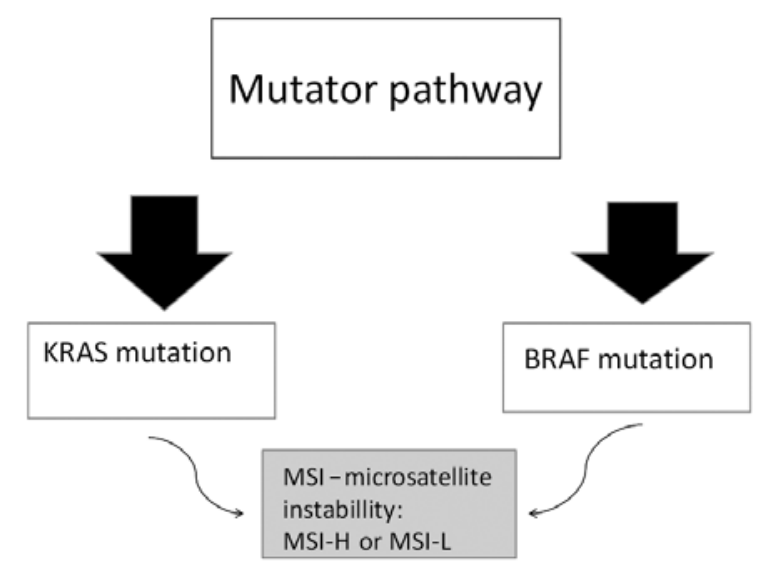

Fig. 3. Mutator pathway methylation takes place at cytosine located 5 ' to guanosine of the dinucleotide sequence CpG. Then cancer-related DNA hypermethylation is present in CG-rich areas called CpG islands. It might result in transcriptional silencing of the gene. CIMP is subdivided into CIMP- high, CIMP-low and CIMP-negative, according to the amount of methylation. CIMP-high exhibits hypermethylation of the promoter region the hMLH-1 (human mutL homolog 1) gene and silences this gene, leading to MSI-H [16]. Additionally to CpG island methylation and MSI-H, a typical feature of the mutator pathway is mutation that activates the BRAF gene. On the other hand, an active RAS-gene stimulates the cell to mitosis and inhibits apoptosis. The next group of mutator pathways are tumors with K-RAS mutation and low levels of $\mathrm{CpG}$ island methylation, e.g. the MSI-L phenotype and some features similar to carcinomas developing through the adenoma-carcinoma sequences [2].

There are much larger unresolved questions in this pathway than in the BRAF mutation pathway. The substantial MGMT (methylguanine-DNA) inactivation by hypermethylation can support a failure to repair G:C to A:T transition. This highlights the DNA MMR systems and increases the mutation level [2].

Recent studies have shown that the mucosa in a healthy population and in patients with hyperplastic polyps have a normal expression of hMLH1 and hMSH2. A decrease in the expression of hMLH1 and hMSH 2 occurs in patients with adenomas and adenocarcinomas. The decrease in the expression of hMLH1 was observed in patients with microsatellite instability (MSI) in CRC [28]. So, inactivation of the genes taking part in MMR lead to MSI in CRC. A reduction of hMLH1 activity is also related to DNA methylation, as shown by Herman et al. [17].

\section{Inflammatory Processes in Colorectal Cancer}

Inflammatory processes play an important role in the development and progression of invasive colorectal tumors, which additionally are the basis by which we can determine their progression and outcome [18]. The inflammatory process is connected to elevated levels of anti-inflammatory cytokine such as interleukin-4 (IL-4) and pro-inflammatory cytokines such as interleukin-6 (IL-6), interleukin-17 (IL-17), tumor necrosis factor (TNF), interleukin-23 (IL-23), interleukin-12 (IL-12) and interleukin-8 (IL-8). There is also interleukin-10 (IL-10), but researchers' opinions on its role are diverse. Some authors consider it as 
a factor in pro-inflammatory processes and others say it has a suppressive action. IL-6 has a lot very important functions in cancer. It is produced by both tumor and normal cells [19]. It is pleiotropic and demonstrates both a pro- and anti-inflammatory role [20]. IL-6 is regarded as correlated with colon cancer increased incidence. Its level in serum in patients with cancer is higher in comparison to healthy controls. Recent studies have shown that it has a significant but not primary role in the pathogenesis of CRC $[19,20]$. According to published data, macrophages are activated by the tumor cells to produce consistently large amounts of IL-6, which affects the production of IL-10 via the STAT3 signaling pathway [19]. Currently, IL-10 still has a controversial dual role in carcinogenesis. On one hand, it is regarded as an immunosuppressive cytokine that affects tumor growth. On the other hand, there are many studies that confirm IL-10's effectiveness in the host response against cancer [21]. However, all researchers agree that its serum levels are significantly elevated in patients with CRC compared to healthy people and postoperative patients. The role of IL-10 is significant in cancer development and prognosis, which is confirmed by several studies [21-24]. Cecev et al. shows that IL-10 has an effect on the differentiation of cancer. Immunohistochemical studies have shown that the level of IL-10 is higher in well-differentiated and moderately differentiated tumors compared to poorly differentiated ones. Therefore, such findings support the protective role of IL-10 in the development and progression of CRC [23]. On the other hand, in recent research based on the level of IL-10 in all of the 4 stages of the TNM classification of CRC, significant differences were found between patients and healthy individuals. Moreover, colon cancer patients in the fourth stage of the clinical advancement of the disease have a higher level of IL-10 in serum compared to the other 3 stages. The summation of such studies indicates that IL-10 has a pro-inflammatory role that is linked to tumor growth $[21,22]$.

Nayko et al. shows that the sex of the patient and the tumor location within the colon vs. the rectum did not affect the differences in IL-10 serum levels [22]. The effects of IL-10 are affected by other pro-inflammatory cytokines such as IL-12. Their activity is described as antagonistic. The increase in IL-10 is associated with decreased production of IL-12. This behavior was observed in CRC. Recent studies have shown that patients with CRC have decreased levels of IL-12. This cytokine level decreases respectively in the individual clinical stages of the disease. In the fourth stage, the levels are lowest when compared to the other 3 on the TNM scale. IL- 12 is produced mainly by Th 1 cells, and to a lesser extent by B cells, mast cells and macrophages. IL-12 affects the production of cytokines such as TNF-alpha and IFN-gamma. Additionally, IL-10 inhibits the production of the latter by suppression of IL-12 transcription. This last mentioned phenomenon is important, as IL-12, when it is reduced, can be used as a marker predicting the presence of cancer metastases in lymph nodes [24]. The bioactive form of IL-12 is IL-12p70, which consists of 2 subunits, namely p35 and p40. The p40 subunit can combine with a p19 subunit to form IL-23, which, like IL-12p70, affects the activation of memory $\mathrm{T}$ cells to produce IFN-gamma and the production of pro-inflammatory cytokines such as IL-17 by Th17. Recent studies compared the serum levels of IL-23 and IL-17 in patients with CRC who were divided into the 4 stages according to the TNM scale and their post-operative levels. It showed that the level of IL-23 is elevated in patients with CRC compared to healthy people. Additionally, the authors observed that there were no differences between level of IL-23 and IL-17 in different clinical stages and they do not changed after surgery [22].

Another important cytokine in the inflammatory process is IL-4. This anti-inflammatory cytokine is produced mainly by Th-2 cells and its level is significantly higher in patients with CRC compared to healthy subjects [25]. IL-4 inhibits the expression of E-cadherin and carcinoembryonic antigen (CEA). This affects the inhibition of cell-cell adhesion of colon cancer cells. A study conducted by Kanai et al. shows that cancer cells treated with IL-4 levels decreased E-cadherin and CEA molecules compared to untreated cells. Abnormal adhesion is responsible for metastasis and invasion in CRC. However, studies haven't shown that inhibition of adhesion of cancer cells has an effect on metastasis and invasion. Additionally, an imbalance between Th1 and Th2 cytokine levels is important in prognosis. It was found that patients with CRC whose cytokines were produced mainly by Th2 cells predisposes them to liver metastases [26].

Lastly, there is IL- 8 which can be produced by both normal and tumor tissues. It plays an important role in acute and chronic inflammatory processes. The studies show that in colon cancer, there is an over-expression of IL-8 as compared to patients with a normal mucosa. The level of IL-8 is related to tumor differentiation. Significantly higher levels are often observed in poorly-differentiated cancer and moderately-differentiated cancer in comparison to well-differentiated [23]. An interesting study on the expression of IL-8 provided by McLean et al. showed over-expression of IL-8 in CRC and adenoma as well as adenocarcinoma compared to those with a normal mucosa [18]. 


\section{Expression \\ of Cyclooxygenase-2 \\ in Serrated Adenomas, Non-Serrated Adenomas, Hyperplastic Polyps and Colorectal Cancers}

The inflammatory process is reflected by the amount of cytokines and cyclooxygenase- 2 (COX-2) protein [27]. COX-2 is a prostaglandin synthetase enzyme. It is involved in the conversion of arachidonic acid to prostaglandin $\mathrm{H} 2$ $\left(\mathrm{PGH}_{2}\right)$, an important precursor of prostacyclin and thromboxane A2. The expression level of COX-2 is elevated in many cancers (e.g. in gallbladder carcinomas along with increased angiogenesis) [28]. $\mathrm{PGH}_{2}$, the product of COX-2, is converted by prostaglandin E2 synthase into prostaglandin $\mathrm{E} 2\left(\mathrm{PGE}_{2}\right)$, which can stimulate cancer progression. Expression of COX-2 is regulated by IL- $1 \beta$ [26]. IL- $1 \beta$ is a pro-inflammatory cytokine produced by activated macrophages. This interleukin affects the activity of cells including cell proliferation, differentiation and apoptosis.

Many studies have shown a significant increase in COX-2 in patients with CRC [29-31]. It was proved that COX-2 plays an important role in CRC, highlighted by the fact that the regular use of COX-2 inhibitors, such as aspirin, reduces the risk of development of CRC [31]. Immunoexpression of COX-2 does not emerge in normal mucosa and mucosa with hyperplastic polyps, but there is an increase in the expression of COX-2 in adenomas and adenocarcinomas. In these studies, no correlation between the expression of COX-2 and age, sex, location and degree of dysplasia or adenoma size was found. Further studies on the expression of COX-2 in colon neoplasia conducted by Kawasaki and Balbinotti have demonstrated that increased expression of COX-2 is not related to serrated lesions. They showed that COX-2 expression was higher in non-serrated adenomas in comparison to HPP, MP with SSA and adenoma and SSA. The subtypes of non-serrated adenoma such as TVA, VA and TA showed no significant differences in the expression of COX-2. Later it was shown that increased expression of COX-2 in non-serrated adenomas is similar to that of traditional serrated adenomas. Over-expression of COX-2 in colorectal adenocarcinomas did not show any differences related to cancers with or without serrations, but was still higher in comparison to non-serrated adenomas [30, 31].

\section{The Role of Anti- -Inflammatory Agents in Carcinogenesis}

It has been observed that using inhibitors of the COX-2 protein might have benefits in the prevention and treatment of gastrointestinal cancers [32, 33]. COX-2 is the target for non-steroidal anti-inflammatory drugs (NSAIDs) and selective cyclooxygenase-2 (COX-2) inhibitors (COXIBs). The risk of malignancies among the elderly (especially over 75 years of age) significantly increases [34]. Elderly patients also often have several parallel diseases such as arthritis, diabetes, cardiovascular disease or psoriasis. In all of them, chronic inflammation plays a major role, thus anti-inflammatory agents are the basic method of treatment. However, anti-inflammatory therapy may affect the results of cancer therapy [32,35]. Low doses of NSAIDs have a favorable impact on many disease treatments, but it can also have negative effects, such as bleeding from the upper and lower parts of the gastrointestinal tract. The risk also increases with simultaneous use of drugs such as NSAIDs, selective cyclooxygenase-2 inhibitors, and corticosteroids or other anticoagulants. Of note, low doses of aspirin $(<325$ mg per day) are recommended for patients at high risk of developing blood clots, to help prevent heart attacks, strokes, and blood clot formation. In addition, there is growing evidence that long-term aspirin therapy reduces the risk of colon cancer. High doses of aspirin (> $325 \mathrm{mg}$ per day) have analgesic and strong anti-inflammatory effects. Ultimately, individual assessment of the benefits and risks of using NSAIDs is the key to effective treatment $[33,36-38]$.

Kim et al., in their study on mouse models, introduced a new family member, namely COXIB CG100649. It inhibits the activity of both COX-2 and carbonic anhydrase-II-I (I-II-CA). CG100649 inhibits the development of premalignant and malignant colorectal lesions via inhibition of tumor cell proliferation. A long-term safety profile of CG100649 has not yet been established [39]. The use of COX-2 inhibitors also causes serious consequences in the form of cardiovascular events such as hypertension and kidney toxicity. The risk increases in patients with pre-existing risk factors for cardiovascular disease [40].

\section{The Problem of Treatment of Colorectal Cancer in Geriatric Patients}

More than half of the people suffering from a cancer, especially colon cancer, are elderly [34, 41]. The development of cancer increases with age and 
reaches its peak in people over 65 years [42]. Cancer is a leading cause of death in older women and men aged 60-79 years [43]. Geriatric patients are special as, in comparison to younger ones, they may have comorbidities, polypharmacy and reduced physiological tissues and organs reserves. Their treatment is a challenge for the oncologist in selecting appropriate treatment and predicting the long-term treatment results. Older age is also associated with increased toxicity of treatment and its side effects [44, 45]. Yet another problem for oncologists in the management of such patients is the low number of older patients in large clinical trials for the treatment of colorectal cancer, even though they constitute the majority of those suffering. However, in recent years there has been a change in the approach to geriatric patients and they are also included in clinical trials [46]. In addition, nowadays in developed countries, it has been noted that older people with cancer are often in better physical form and they are able to tolerate even the most intensive treatment. However, in early stages of the disease, clinical dilemmas appear regarding the ability of elderly patients to undergo successful surgical and therapeutic treatment and the evaluation of the risk-benefit ratio of adjuvant chemotherapy giving the benefit of prolonged life free from disease. According to Foster et al., oncologists planning the treatment of older people suffering from colon cancer are more likely to choose conservative treatment in these patients instead of the standard treatment of cancer, despite the patient's good condition [44]. The author also draws attention to the need for a more profound look at geriatric patients' oncologists when choosing a cancer treatment in which age is not a key determinant of therapy. For clinical practice, applying a method of comprehensive geriatric assessment (CGA) may facilitate this process and improve longevity estimates, which may have an impact on the treatment of the patient [44]. Geriatric assessment (CGA) is a multidisciplinary assessment of the functional status of the patient, comorbidities, mental status, social support, cognitive function and nutritional status. Nevertheless, this is a very time-consuming method, so the alternative simplified method of geriatric assessment is often used by oncologists, which is based on the division of patients into "fit" and "frail" categories $[44,45]$. A patient referred to as "fit" is in good condition and has limited comorbidities and geriatric syndromes. They are at increased risk of cancer incidence and mortality due to cancer. Patients referred to as "frail" are in poor general condition and have many comorbidities and geriatric syndromes. These patients have a limited life expectancy due to other diseases and are more susceptible to morbidity and mortality from other comorbidities, and not only the cancer. And an approach to therapy has been proposed with these two different categories, with more aggressive treatment for "fit" patients and more conservative therapies for "frail" patients. Dotan et al. gave his analysis of the benefits of surgery, adjuvant chemotherapy and aggressive surveillance in "fit" older patients with early stages of colorectal cancer [45]. The results were comparable to younger patients. The decision is more complicated for older patients assigned to the "frail" category, for whom a treatment strategy should be determined by a joint decision of the patient and oncologist. Treatment of elderly patients with metastatic colorectal cancer requires careful rethinking of the strategy of treatment, overall, the demonstrated clinical benefit at the expense of increased toxicity when the agents are approved for use in the elderly population. The coefficient of risk/benefit of treatment should be considered prior to treatment [45]. Additional clinical trials targeting the older population are needed to increase our knowledge of the optimal management of elderly patients with colorectal cancer, and to be able to know the impact of treatment of comorbidities on oncological treatment for "frail" patients.

\section{Final Remarks}

In spite of numerous, intensive studies and defining CRC as one of the most well-known types of cancer, the pathogenesis of CRC is still not fully understood. Further specific and particularly detailed studies should allow us to describe more and more accurate pathways including genetic, environmental and epigenetic factors. It has been determined that the key phenomena are genetic and inflammatory processes. The well-known genetic basis for carcinogenesis in CRC includes chromosomal changes characteristic for the CIN pathway, which correlates to specific and well-defined genetic alterations (such as APC, K-RAS, DCC and p53a), and genomic instability characteristic of the mutator pathway, focused on KRAS and BRAF mutations. Another aspect of the carcinogenesis is the impact of inflammation on CRC, with detailed studies on the elevated levels of cytokines such as IL-6, IL-4, IL-8, IL-12, IL-17, TNF, IL-23 and IL-10, which some authors consider a pro-inflammatory factor and others as a suppressive factor. Particularly noteworthy are recent studies in which it has been shown that different adenomas are characterized by different processes of carcinogenesis, which was demonstrated by COX-2 expression in various polyps. Understanding the mechanism of inflammatory processes in CRC and combining it with genetic alterations should allow for effective and targeted treatment. 


\section{References}

[1] Spann S, Levin B, Rozen P, Young G: Colorectal cancer: How big is the problem, why prevent it and how might it present? In: Colorectal cancer in clinical practice: prevention, early detection and management. Eds.: Rozen P, Young G, Levin B. Martin Dunitz, London 2002, 1-13.

[2] Stefanius K: Colorectal carcinogenesis via serrated route. Acta Univ Oul D 2011, 1091.

[3] Balkwill F, Mantovani A: Inflammation and cancer: back to Virchow? Lancet 2001, 357, 539-545.

[4] Morrison WB: Inflammation and Cancer: A Comparative View. J Vet Intern Med 2012, 26, 18-31.

[5] Goswami B, Rajappa M, Sharma M: Inflammation: its role and interplay in the development of cancer, with special focus on gynecological malignancies. Int J Gynecol Cancer 2008, 18, 591-599.

[6] Coussens LM, Werb Z: Inflammation and cancer. Nature 2012, 420, 860-867.

[7] Jemal A, Siegel R, Ward E, Murray T, Xu J, Smigal C, Thun MJ: Cancer Statistics. CA Cancer J Clin 2006, 56, $106-130$.

[8] Kwong LN, Dove WF: APC and its modifiers in colon cancer. Adv Exp Med Biol 2009, 656, 85-106.

[9] Longacre TA, Fenoglio-Preiser CM: Mixed hyperplastic adenomatous polyps/serrated adenomas. Am J Surg Pathol 1990, 14, 524-537.

[10] Torlakovic EE, Gomez JD, Driman DK: Sessile Serrated Adenoma (SSA) vs. Traditional Serrated Adenoma (TSA). Am J Surg Pathol 2008, 32, 21-44.

[11] Valerie P, Bauer MD, Harry T: Management of Serrated Adenomas and Hyperplastic Polyps. Clin Colon Rectal Surg 2008, 21, 273-279.

[12] Fearon ER, Vogelstein B: A Genetic model for Colorectal Tumorigenesis. Cell 1990, 61, 759-767.

[13] Sandmeier D, Benhattar J, Martine P: Serrated polyps of the large intestine: a molecular study comparing sessile serrated adenomas and hyperplastic polyps. Histopathology 2009, 55, 206-213.

[14] Krasinskas AM: EGFR Signalling in colorectal carcinoma. Patholog Res Int 2011.

[15] Itoh F, Imai K: Genetic diagnosis of colorectal cancer. Hokkaido Iqaku Zasshi 1996, 71, 9-14.

[16] Weisenberger HT: Genome-scale analysis of aberrant Dna methylation in colorectal cancer. Genome Res 2012, $22,271-282$.

[17] Herman JG, Umar A, Polyak K, Graff JR, Ahuja N, Issa JP, Markowitz S, Willson JK, Hamilton SR, Kinzler KW, Kane MF, Kolodner RD, Vogelstein B, Kunkel TA, Baylin SB: Incidence and functional consequences of hMLH1 promoter hypermethylation in colorectal carcinoma. Proc Natl Acad Sci U S A 1998, 95, 6870-6875.

[18] McLean MH, Murray GI, Stewart KN, Norrie G, Mayer C: The Inflammatory Microenvironment in Colorectal Neoplasia. PLoS ONE 2011, 6, e15366.

[19] Herbeuval JP, Lelievre E, Lambert C, Dy M, Genin C: Recruitment of STAT3 for Production of IL-10 by Colon Carcinoma Cells Induced by Macrophage-Derived IL-6. J Immunol 2004, 172, 4630-4636.

[20] Becker C, Fantini MC, Wirtz S: IL-6 Signaling Promotes Tumor Growth in Colorectal Cancer. Cell Cycle 2005, 4, $217-220$.

[21] Stanilov N, Stankova N, Miteva L, Jovchev J, Deliyski T, Stanilova S: Role of IL-12P40 and IL-10 in progression of colorectal cancer. TJS 2010, 8, 132-136.

[22] Stanilov N, Miteva L, Deliysky T, Jovchev J, Stanilova S: Advanced Colorectal Cancer Is Associated With Enhanced IL-23 and IL-10 Serum Levels. Labmedicine 2010, 41, 159-163.

[23] Cacev T, Radosevic S, Krizanac S, Kapitanović S: Influence of interleukin-8 and interleukin-10 on sporadic colon cancer development and progression. Carcinogenesis 2008, 29, 1572-1580.

[24] O’Hara R J, Greenman J, MacDonald AW, Gaskell KM, Topping KP, Duthie GS, Kerin MJ, Lee PW, Monson JR: Advanced colorectal cancer is associated with impaired interleukin-12 and enhanced interleukin-10 production. Clin Cancer Res 1998, 4, 1943-1948.

[25] Galon J, Costes A, Sanchez-Cabo F, Kirilovsky A, Mlecnik B, Lagorce-Pagès C, Tosolini M, Camus M, Berger A, Wind P, Zinzindohoué F, Bruneval P, Cugnenc PH, Trajanoski Z, Fridman WH, Pagès F: Type, density, and location of immune cells within human colorectal tumors predict clinical outcome. Science 2006, 313, $1960-1964$.

[26] Kanai T, Watanabe M, Hayashi A, Nakazawa A, Yajima T, Okazawa A, Yamazaki M, Ishii H, Hibi T: Regulatory effect of interleukin-4 and interleukin-13 on colon cancer cell adhesion. Br J Cancer 2000, 82, 1717-1723.

[27] Wiiliam CS, Mann M, Dubois RN: The role of cycloxygenases in inflammation, cancer and development. Oncogene 1999, 18, 7906-7916.

[28] Legan M: Cyclooxygenase-2, p53 and glucose transporter-1 as predictors of malignancy in the development of gallbladder carcinomas. Bosn J Basic Med Sci 2010, 10, 192-196.

[29] Gustafsson Asting A, Caren H, Andersson M, Lönnroth C, Lagerstedt K, Lundholm K: COX-2 gene expression in colon cancer tissue related to regulating factors and promotor methylation status. BMC Cancer 2011, 11, 238.

[30] Balbinotti RA, Ribeiro U, Saka P, Safatle-Ribeiro AV, Balbinotti SS, Scapulatempo C, Alves VA, Corbett CE, Carrilho FJ: hMLH1, hMSH2 and Cyclooxygenase-2 (Cox-2) in Sporadic Colorectal Polyps. Anticancer Res 2007, 27, 4465-4472.

[31] Kawasaki T, Nosho K, Ohnishi M, Suemoto Y, Glickman JN, Chan AT, Kirkner GJ, Mino-Kenudson M, Fuchs CS, Ogino S: Cyclooxygenase-2 overexpression is common in serrated and non-serrated colorectal adenoma, but uncommon in hyperplastic polyp and sessile serrated polyp/adenoma. BMC Cancer 2008, 8, 33.

[32] Menter DG, Schilsky RL, DuBois RN: Cyclooxygenase-2 and cancer treatment: understanding the risk should be worth the reward. Clin Cancer Res 2010, 16, 1384-1390.

[33] Romagnolo DF, Papoutsis AJ, Selmin O: Nutritional targeting of cyclooxygenase-2 for colon cancer prevention. Inflamm Allergy Drug Targets 2010, 9, 181-191. 
[34] Pelzer O, Kielan W: Risk analysis for the surgical treatment of colorectal cancer in elderly patients undergoing scheduled and urgent interventions. Pol Przegl Chir 2014, 86, 61-67.

[35] Rayburn ER, Ezell SJ, Zhang R: Anti-Inflammatory Agents for Cancer Therapy. Mol Cell Pharmacol 2009, 1, 29-43.

[36] Peura DA, Wilcox CM: Aspirin and proton pump inhibitor combination therapy for prevention of cardiovascular disease and Barrett's esophagus. Postgrad Med 2014, 126, 87-96.

[37] Sostres C, Lanas A: Gastrointestinal effects of aspirin. Nat Rev Gastroenterol Hepatol 2011, 8, 385-394.

[38] Lanas A: Gastrointestinal bleeding associated with low-dose aspirin use: relevance and management in clinical practice. Expert Opin Drug Saf 2011, 10, 45-54.

[39] Kim SH, Margalit O, Katoh H, Wang D, Wu H, Xia D, Holla VR, Yang P, DuBois RN: CG100649, a novel COX-2 inhibitor, inhibits colorectal adenoma and carcinoma growth in mouse models. Invest New Drugs 2014, PMID: 25085205.

[40] Cooper K, Squires H, Carroll C, Papaioannou D, Booth A, Logan RF, Maguire C, Hind D, Tappenden P: Chemoprevention of colorectal cancer: systematic review and economic evaluation. Health Technol Assess 2010, $14,1-206$.

[41] Lin LL, Hahn SM: Combined modality therapy in the elderly population. Curr Treat Options Oncol 2009, 10, 195-204.

[42] U.S. Census Bureau: National Population Projections. Percent Distribution of the Projected Population by Selected Age Groups and Sex for the United States: 2010 to 2050. http://www.census.gov/population/www/projections/summarytables.html, accessed 2009.

[43] Jemal A, Siegel R, Xu J, Ward E: Cancer statistics 2010. CA Cancer J Clin 2010, 60, 277-300.

[44] Foster JA, Salinas GD, Mansell D, Williamson JC, Casebeer LL: How Does Older Age Influence Oncologists' Cancer Management? The Oncologist 2010, 15, 584-592.

[45] Dotan E, Browner I, Hurria A, Denlinger C: Challenges in the Management of Older Patients with Colon Cancer. J Natl Compr Canc Netw 2012, 10, 213-225.

[46] Lewis JH, Kilgore ML, Goldman DP, Trimble EL, Kaplan R, Montello MJ, Housman MG, Escarce JJ: Participation of patients 65 years of age or older in cancer clinical trials. J Clin Oncol 2003, 21, 1383-1389.

\section{Address for correspondence:}

Łukasz Szylberg

Department of Clinical Pathomorphology

Collegium Medicum in Bydgoszcz

Nicolaus Copernicus University in Toruń

Skłodowskiej-Curie 9

85-094 Bydgoszcz

Poland

Tel.: +48525854200

E-mail: 1.szylberg@gmail.com

Conflict of interest: None declared

Received: 15.01.2014

Revised: 7.09.2014

Accepted: 26.09.2014 